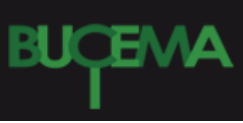

Bulletin du centre d'études médiévales d'Auxerre | BUCEMA

Hors-série $n^{\circ} 3 \mid 2010$

Présentation et mise en valeur des sites archéologiques religieux en milieu urbain

\title{
La crypte archéologique de Saint-Germain d'Auxerre (Yonne)
}

\section{Christian Sapin et Daniel Massicard}

\section{(2) OpenEdition \\ Journals \\ Édition électronique \\ URL : https://journals.openedition.org/cem/11371 \\ DOI : $10.4000 /$ cem. 11371 \\ ISSN : 1954-3093 \\ Éditeur \\ Centre d'études médiévales Saint-Germain d'Auxerre}

Référence électronique

Christian Sapin et Daniel Massicard, «La crypte archéologique de Saint-Germain d'Auxerre (Yonne) », Bulletin du centre d'études médiévales d'Auxerre / BUCEMA [En ligne], Hors-série n 3 | 2010, mis en ligne le 18 mars 2010, consulté le 03 mars 2023. URL : http://journals.openedition.org/cem/11371 ; DOI : https://doi.org/10.4000/cem.11371

Ce document a été généré automatiquement le 3 mars 2023.

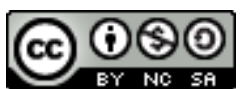

Creative Commons - Attribution - Pas d'Utilisation Commerciale - Partage dans les Mêmes Conditions 4.0 International - CC BY-NC-SA 4.0

https://creativecommons.org/licenses/by-nc-sa/4.0/ 


\title{
La crypte archéologique de Saint- Germain d'Auxerre (Yonne)
}

\author{
Christian Sapin et Daniel Massicard
}

1 Le chemin qui conduira à la réalisation dela crypte archéologique de Saint-Germain d'Auxerre commence en 1986. Elle constituera, avec les publications, un des termes du vaste projet initié dans le cadre d'un programme de recherche CNRS croisé avec un plan patrimonial. Le programme de recherche n'était, à l'origine, qu'une enquête historique et archéologique, avec le souci néanmoins de s'insérer dans une réflexion sur la présentation du patrimoine de l'abbaye (restauration des bâtiments dont la crypte carolingienne et l'aménagement du musée dans les salles), sans prétendre à l'aménagement d'une crypte archéologique. Ces éléments seront déterminants dans le montage financier de l'ensemble de l'opération à travers trois conventions croisées entre État (Monuments historiques, Direction des Musées de France et Service départemental de l'Archéologie), la ville d'Auxerre et les collectivités territoriales.

\section{Le programme de recherche sur Saint-Germain d'Auxerre.}

2 Ce programme initié en 1985-1986 aurait pu s'appeler De la villa à l'abbaye ou De l'Histoire à l'archéologie.

3 En effet, si l'on connaissait l'évolution urbaine dans ses grandes lignes, celle de l'abbaye était tributaire d'une histoire basée sur des compilations et sans reconnaissances sérieuses de terrain. Au moment où l'équipe de Georges Duby du Collège de France (avec en particulier Dominique Iogna-Prat et Guy Lobrichon) étudiait systématiquement les œuvres produites à Auxerre au IX siècle, il s'agissait de comprendre la réalité matérielle du lieu de production. Comment trois générations de moines lettrés avait pensé l'espace et plus encore comment était-on passé d'un simple oratoire où était inhumé l'évêque de la ville Germain à une abbaye qui allait avoir un retentissement européen? Ce travail sur la longue durée passait par une enquête historique des archives, une relecture des sources (avec J.-C. Picard) jusqu'à la 
transformation de l'abbaye en hôpital au début du XIX siècle. En parallèle, de nouveaux relevés et plans sur le terrain préparaient les campagnes archéologiques qui allaient se dérouler sur le site de 1989 à 1999. Outre une meilleure connaissance des cryptes carolingiennes et de leurs peintures, des investigations sur tout le cloître et aux abords de l'abbatiale, il s'agissait de comprendre la partie occidentale de l'édifice, détruite en 1812. En effet, l'analyse des documents iconographiques avait poussé nos prédécesseurs à voir dans cette partie disparue les vestiges d'une avant-nef carolingienne. Cette interprétation des années 1950-1960, influencée par les grands sites allemands désormais mieux connus, demandait une connaissance du terrain et de véritables fouilles. Durant dix ans, ces travaux programmés sur $750 \mathrm{~m}^{2}$ allaient se conjuguer avec un vaste plan de travaux dans une perspective patrimoniale qui devait restructurer l'abbaye depuis le départ de l'hôpital en 1968. La dimension atteinte par ces travaux et les bonnes conditions d'interventions n'auraient pu se faire sans ce double cadre scientifique (CNRS, Ministère de la Culture) et institutionnel (Ville, état et collectivités). Ce sont ces mêmes conditions favorables qui ont permis progressivement, d'introduire l'idée et la réalisation d'une crypte archéologique et de mener rapidement à bien les publications qui ont suivi dès 1999-2000 ${ }^{1}$.

4 Un site Web du ministère de la Culture complète ce renouveau dans la perception d'un site majeur et méconnu [http://www.auxerre.culture.gouv.fr/].

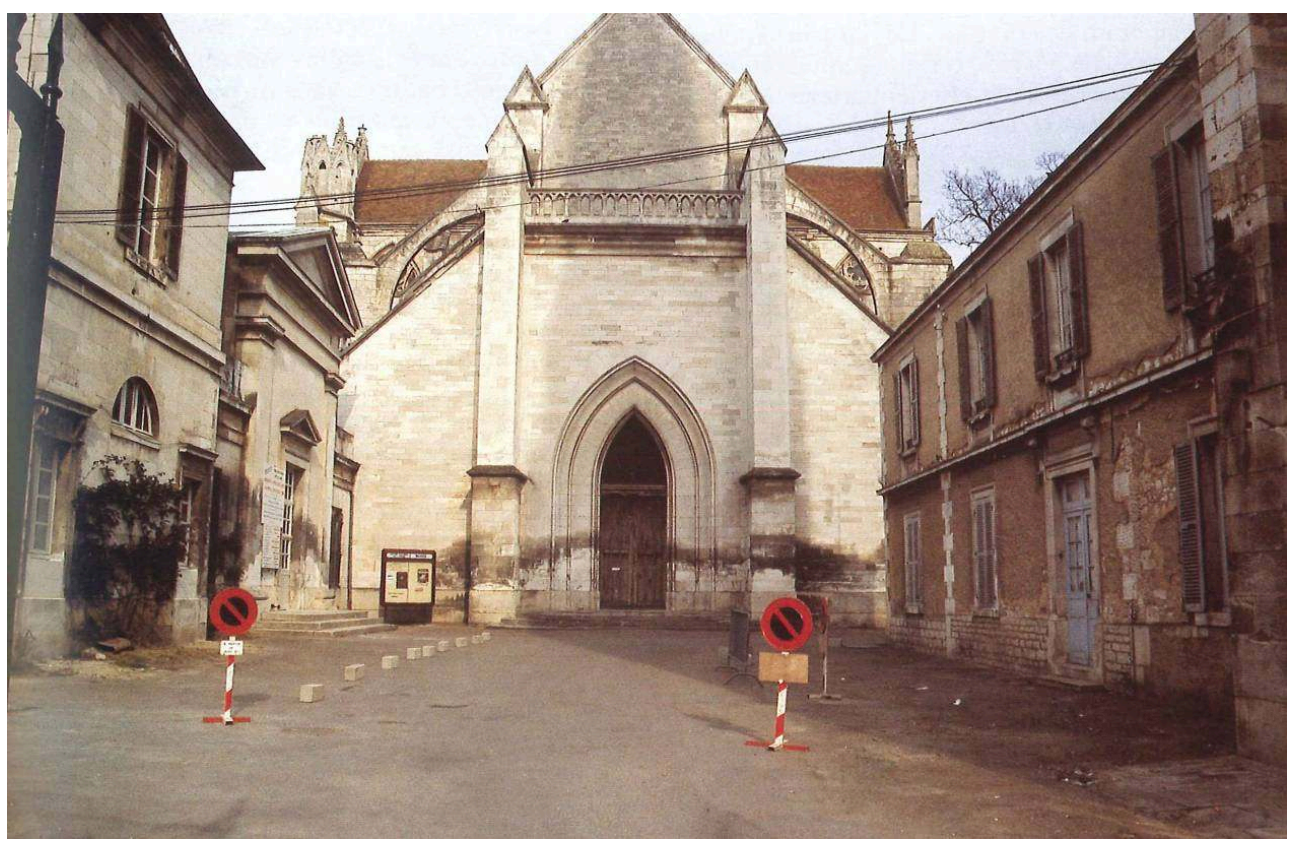

Fig. 1 - Avant-nef, emplacement avant le début des fouilles en 1989 (cl. CEM). 


\title{
Le programme patrimonial et la restauration de l'abbaye 1981-2001
}

\author{
Restauration et aménagement d'un monument historique ; création \\ d'un musée, d'un centre de recherche ; le point de vue du maître \\ d'ouvrage :
}

5 Au début des années 1980, la municipalité d'Auxerre a le projet de réhabiliter l'ancienne abbaye Saint-Germain, témoignage du rayonnement intellectuel de l'École d'Auxerre entre le $\mathrm{IX}^{\mathrm{e}}$ et le $\mathrm{XI}^{\mathrm{e}}$ siècle. Le projet est, d'une part, de restaurer un monument historique prestigieux, un des plus anciens de la ville encore visible et, d'autre part, d'y abriter puis d'y présenter les collections du musée. Au-delà de ce double objectif, la municipalité considère ce monument comme le joyau de son patrimoine, capable d'attirer un tourisme culturel et, sur Auxerre, susceptible de proposer aux Icaunais des expositions permanentes et temporaires et des manifestations culturelles à rayonnement régional, voire national.

6 La question qui nous est posée est la suivante : en quoi les recherches archéologiques inhérentes à ce type d'opération ont contribué à redimensionner le projet initial ?

7 Précisons que les travaux comme les recherches archéologiques se sont faits dans le cadre de conventions successives :

81 - Une première convention (1982-1986) a été signée entre l'État et la ville d'Auxerre pour régler deux urgences :

- mettre à l'abri les collections du musée qui, depuis une dizaine d'années, étaient dispersées dans plusieurs sites de la ville, sans aucune protection d'où le projet de réaliser (et c'est ce qui sera fait en premier) les réserves du musée.

- racheter, sur l'hôpital qui venait de quitter les lieux, ses locaux, pour maîtriser l'ensemble des bâtiments.

La municipalité s'est ensuite fixée trois objectifs :

- Restaurer dans sa totalité l'ensemble des bâtiments de l'abbaye.

- Aménager les salles dédiées à la préhistoire, à la période gallo-romaine et à la période médiévale ainsi que les salles dédiées à la peinture.

- Aménager les locaux de services (conservation, entrée, gardiennage, circulation, librairie etc.).

102 - Une $2^{\text {ème }}$ convention( 1986-1992) est construite à partir des capacités financières de quatre partenaires de l'opération: l'État pour $50 \%$; la ville d'Auxerre et la Région Bourgogne pour $50 \%$; le département de l'Yonne pour un montant de 5335000 euros (35 millions de francs) affectés à quatre postes :

- la restauration des bâtiments ;

- l'aménagement des salles attribuées à la préhistoire et à la période médiévale ;

- les fouilles archéologiques ;

- l'accueil-sécurité.

11 L'absence d'expérience des services de la ville sur ce type de dossier, la définition imprécise, sur le plan technique, des postes, ont conduit à ce que soit privilégiée la restauration des bâtiments aux dépends de l'aménagement des salles. 
12 La $2^{\text {ème }}$ convention s'achève sans que les salles médiévales aient pu être aménagées, le coût de la restauration des bâtiments s'étant révélé très supérieur aux estimations inscrites dans la convention.

Seul le chantier de fouilles archéologiques a été réalisé dans les temps et crédits impartis.

Tant en termes de méthode que de résultats, le travail des chercheurs du CNRS a influencé la rédaction de la $3^{\text {ème }}$ convention et va, à terme, modifier sensiblement l'aménagement du musée.

3 - La $3^{\text {ème }}$ convention (1994-2001)

complexité des opérations à réaliser, liée à l'aménagement des salles médiévales à lamise en valeur des cryptes historiques et l'aménagement de la crypte archéologique, conduit la ville à s'entourer d'experts : muséographes, programmiste, historiens, etc.

17 L'équipe de chercheurs du CNRS, qui deviendra quelques années plus tard le Centre d'études médiévales, devient un partenaire à part entière de la convention, au même titre que la DRAC, l'ACMH et les représentants des collectivités territoriales.

18 L'intérêt de leur recherche sur l'abbaye, les propositions de fouilles archéologiques autour de l'église et dans les cryptes, le parrainage de deux médiévistes prestigieux (G. Duby et J. Le Goff), avait conduit les élus à inscrire, dans la première convention, un premier volet de fouilles : le programme était modeste ; il représentait un million de francs (152 450 euros) sur une convention de 35 millions de francs (5 335000 euros).

19 Quelques élus, au conseil municipal et dans la presse, s'interrogent, de façon récurrente, sur le bien-fondé de ces fouilles. La critique est d'autant plus aisée que la lecture des vestiges est loin d'être immédiate pour le profane.

Pourtant, compte tenu de la qualité scientifique des travaux qui ont été menés, de l'intérêt qu'on suscité, peu à peu, la mise au jour des fondations de l'avant-nef de l'église de l'abbaye, les chercheurs se sont implantés sur le site:

- implantation intellectuelle puisque les chercheurs travaillent en continu avec les services de la ville où ils jouent aussi le rôle de conseil dans l'aménagement des salles médiévales ;

- implantation physique puisque la ville leur a attribué des locaux : hébergement pour les chercheurs, labo, salle de réunion, etc.

21 Leur présence a ouvert de nouvelles perspectives : la réalisation d'un musée d'art et d'histoire était légitimée par le haut, d'autant que les chercheurs avaient créé une structure permanente, le Centre d'études médiévales, regroupant quelques 120 chercheurs européens et américains, qui organisera dans les années 1990 des colloques et des expositions prestigieuses.

En conclusion on peut affirmer que les recherches archéologiques ont réorienté le projet initial.

23 Le projet de la municipalité était d'installer dans l'ancienne abbaye un musée d'art et d'histoire. Or, a été réalisé un musée d'histoire; les découvertes archéologiques ont réorienté le projet défini en 1981 et la création, dans l'ancien espace de l'avant-nef, d'une crypte à caractère pédagogique, a contribué à l'abandon de l'aménagement des salles affectées au département beaux-arts; aujourd'hui elles ne sont pas encore programmées.

24 Mais l'apport des chercheurs est allé au-delà de la mise en valeur des fouilles : intégrés à l'équipe des maîtres d'œuvre, en tant que responsable du chantier archéologique, ils 
sont devenus le conseiller du maître d'ouvrage dans l'aménagement des salles médiévales du musée.

\section{Le projet et la réalisation du sous-sol archéologique}

Plusieurs éléments peuvent être retenus dans ce qui a été déterminant dans les choix et la réalisation du sous-sol archéologique de l'avant-nef proprement dite.

Le fait que les premiers sols ou niveaux archéologiques intéressants à présenter se trouvaient à $-2,50 \mathrm{~m}$ du sol actuel constituait un des facteurs les plus favorables au projet; de même que la bonne conservation des vestiges (sur près de deux mètres, bases de piliers, sols) ne pouvait qu'encourager un projet de présentation. En outre, il apparaissait rapidement qu'un tel projet en surface pouvait permettre de marquer la différence entre l'avant-nef historique, l'ancienne nef romane et la nef, tout en redonnant une dimension à l'ensemble de l'abbatiale. Enfin un accès pouvait être facilement réalisé depuis la nef conservée permettant de circuler dans l'espace archéologique au même niveau (celui du xIV ${ }^{e}$ siècle), et d'apercevoir sous le caillebotis les vestiges plus anciens (fig. 2).

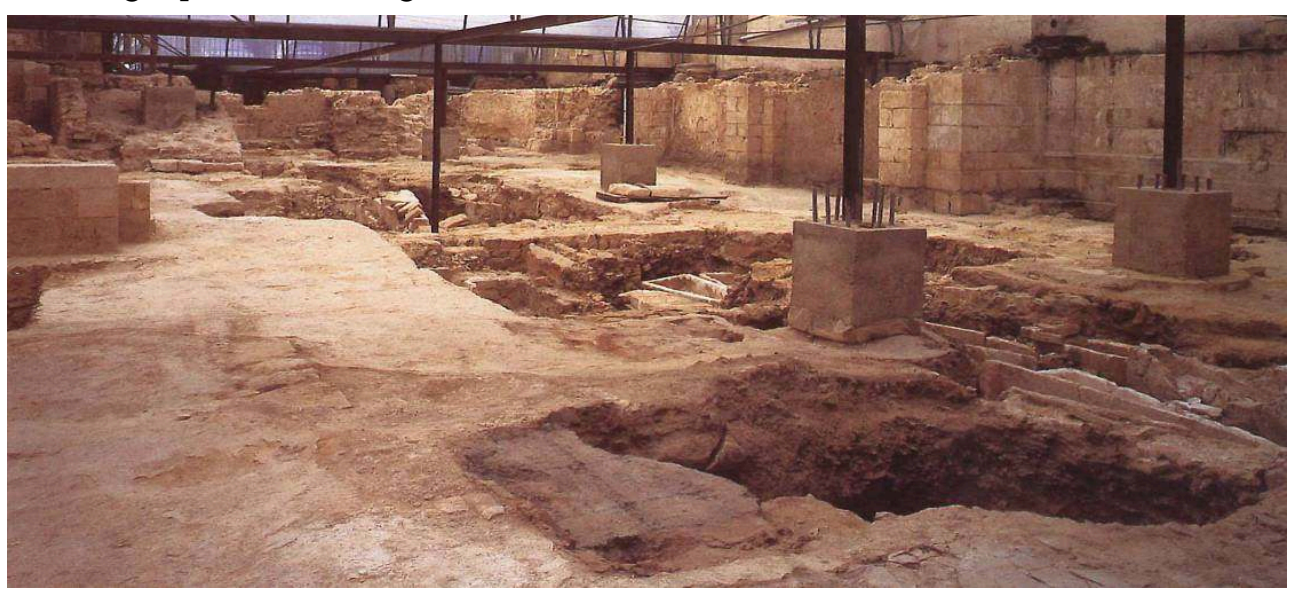

Fig. 2 - Avant-nef en cours de fouille, vue générale. (cl. G. Plouvier).

Ce nouvel espace pouvait constituer un lieu d'attente et de visite pour les groupes, nombreux, qui attendaient à chaque demi-heure les visites guidées de la crypte carolingienne et de ses peintures. En effet à la même époque, les études sanitaires démontraient que pour une bonne conservation des peintures les groupes de visites devaient être limités à moins de trente personnes avec un temps entre chaque visite.

Alors que les fouilles se poursuivaient, émergeait ainsi rapidement (en 1995) l'idée d'un concours d'architecte pour cet espace qui ne dépendait pas nécessairement de l'architecte des Monuments historiques qui avait à sa charge l'abbatiale. Ce fut le choix du Maire et du Conseil municipal d'Auxerre. Il était cependant nécessaire d'avoir l'avis de l'Inspection des Monuments historiques (1997) pour la démolition d'une construction basse établie entre 1850 et 1910 sur l'emplacement du bas-côté sud. Un sondage avait aisément, permis de montrer que les structures archéologiques bien conservées de l'ancienne avant-nef se trouvaient également sous cette maison. Sa démolition réalisée et la fouille poursuivie, il était possible d'affiner le projet avec l'équipe qui avait emporté le concours (Agence Novembre). 


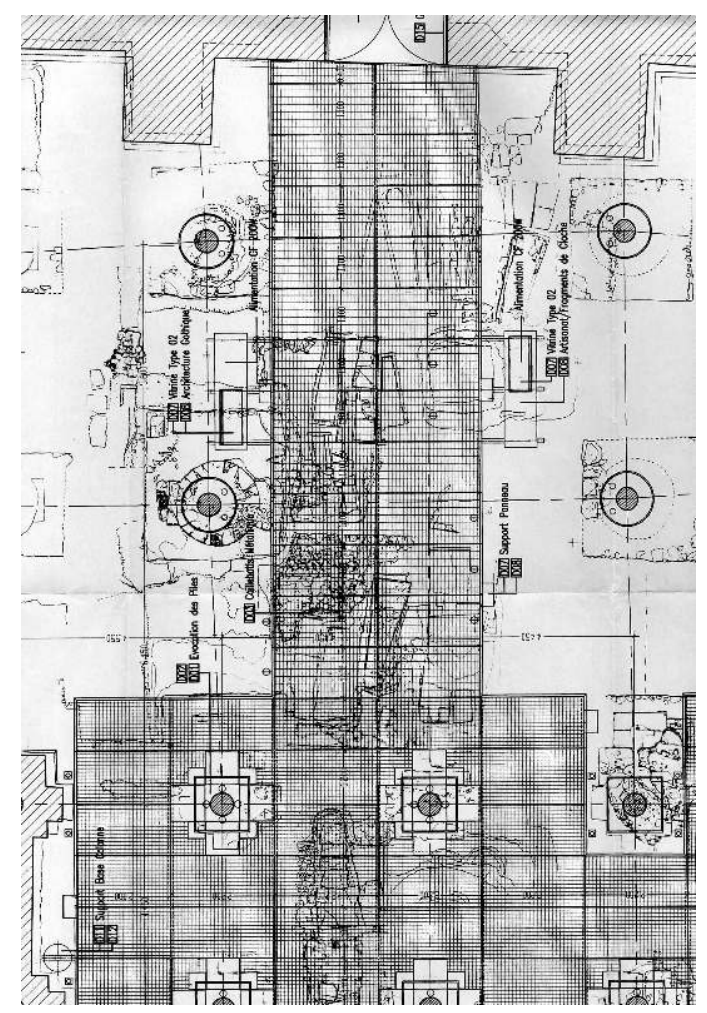

Fig. 3 - Projet du « cabinet Novembre », 2008.

Des choix devaient être faits sur la présentation des murs des piliers en partie démolie, de la circulation du public, etc. Les archéologues ont été constamment associés à cette réflexion ainsi que le conservateur du musée responsable du site monumental. D'autres sites furent visités en France et à l'étranger. Il fut ainsi décidé de marquer à la surface du sol les structures que le public découvrirait dans le sous-sol archéologique, et dans celui-ci de restituer gardeur nature (en matériaux légers et réversibles) trois des piliers composés qui séparaient l'ancienne nef de l'avant-nef. Les traces des autres piliers restaient visibles au sol, seul leurs contours devaient être marqués par des matériaux différents. C'est un système de caillebotis qui fut adopté sur l'ensemble de la surface de la zone archéologique où le public avait accès. Cependant, il a fallu discuté quelque fois fermement sur l'emplacement des supports comme sur la dimension des piliers modernes pour la dalle avec les ingénieurs responsables. Il s'agissait là d'un véritable investissement (de temps) pour l'archéologue et son équipe, soucieux depuis le projet jusqu'au DCE (Document de consultation des entreprises) en 1998, de voir une réalisation conforme à l'intérêt scientifique du site et compréhensible par un grand public. La réalisation des travaux qui demandait des protections provisoires de la fouille (bidim, sable, plancher) exigeait également un suivi constant durant deux ans. Certaines zones ont ainsi été rebouchées; d'autres gardées en réserves archéologiques. Les éclairages ont été pensés en fonction de la hiérarchie des structures dans l'histoire du site, des pupitres d'explication avec des dessins ont été prévus en plusieurs endroits. Certains éléments ont été sous-estimés comme les effets de l'humidité sur des secteurs mal drainés ou ventilés. Le chantier a pris un an de retard par rapport à la programmation envisagée et c'est en juin 1999 que l'avant-nef archéologique a été inaugurée, en même temps qu'une exposition et qu'un colloque faisaient le point sur les avant-nefs en Europe. 


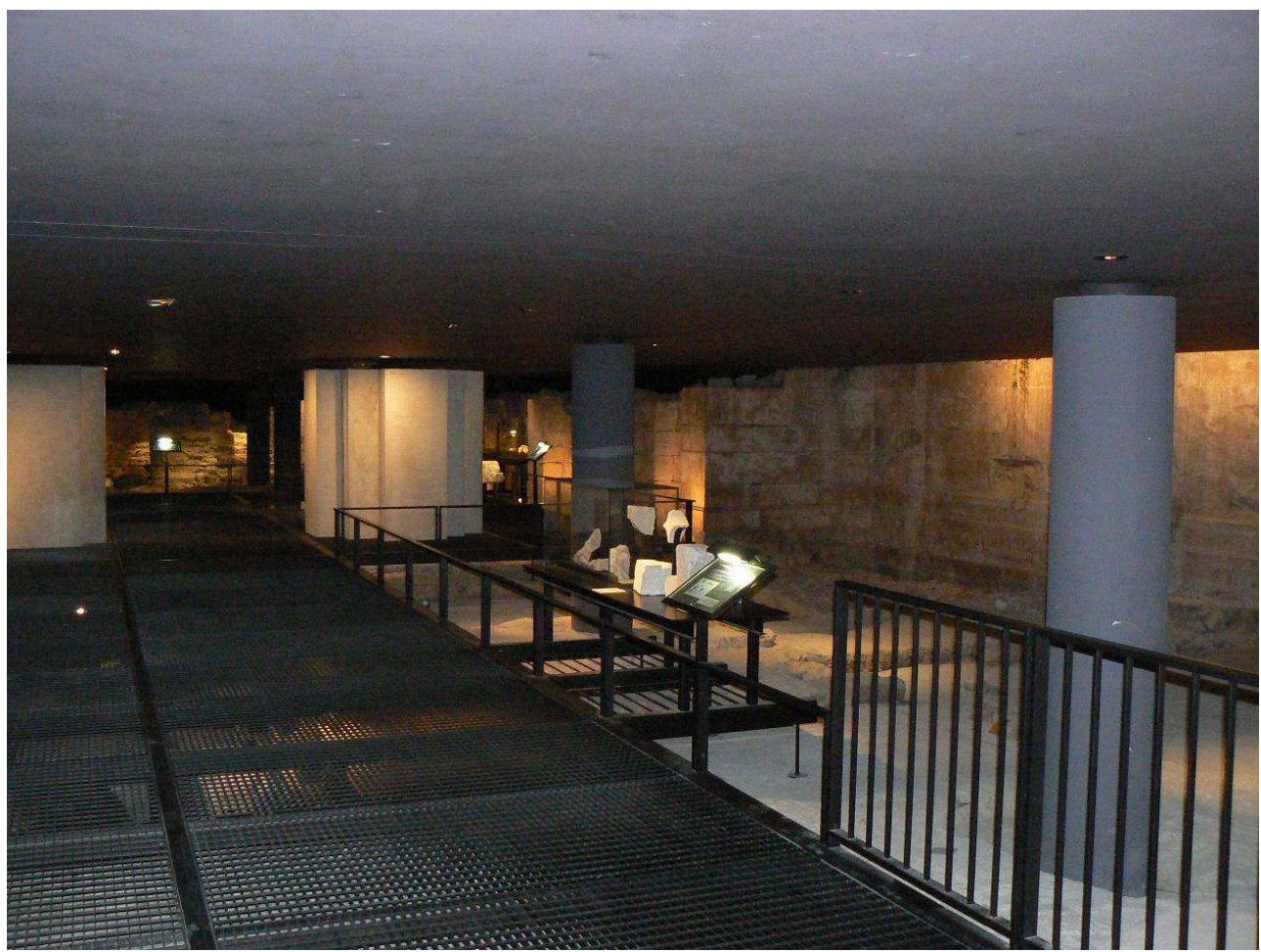

Fig. 4 et 5 - Avant-nef aménagée (cl. CEM).

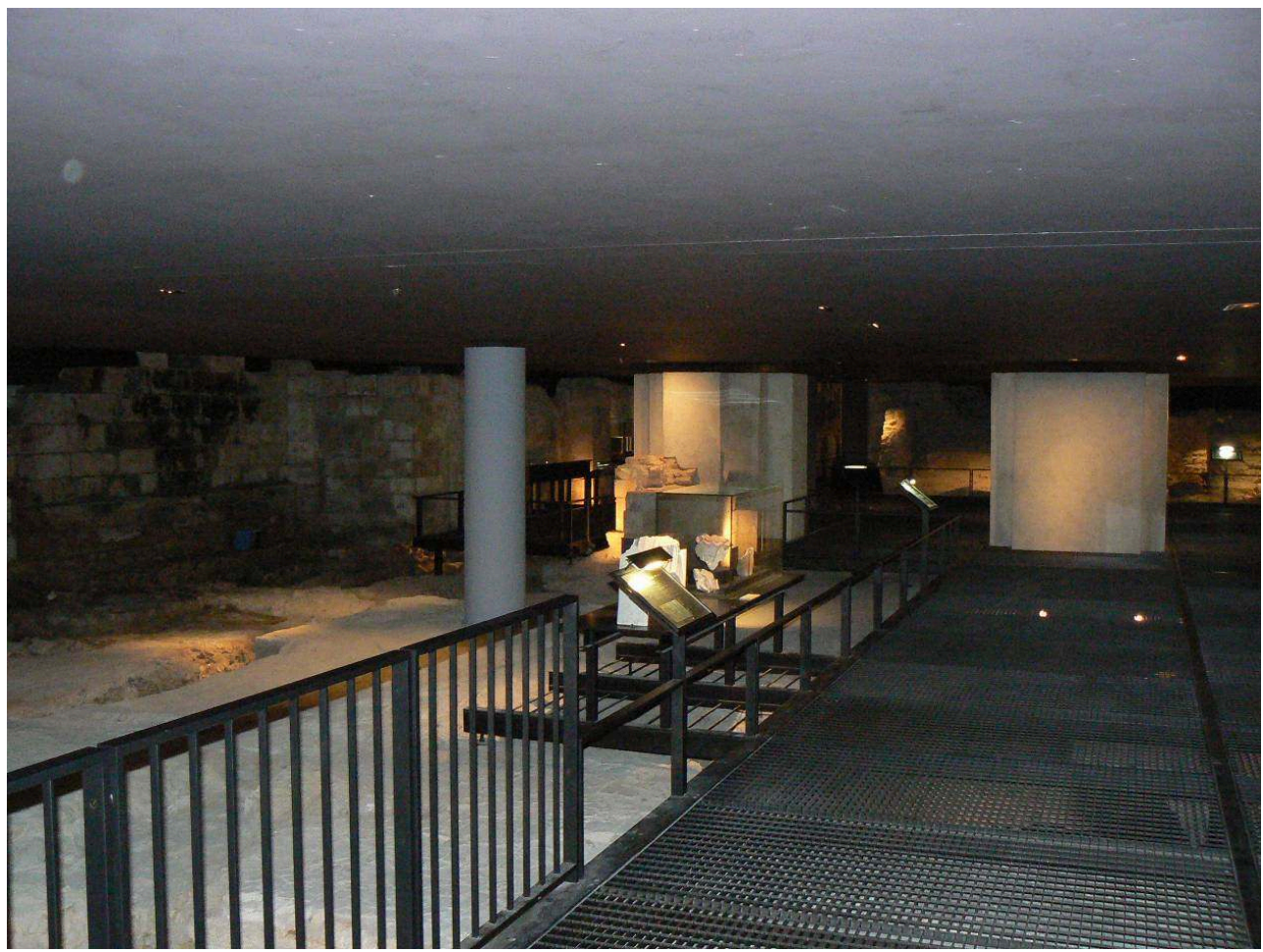

En conclusion, on peut retenir que le sous-sol archéologique réalisé à Saint-Germain d'Auxerre, en dépit de ses difficultés (problème de maintenance) ou erreurs (zones humides insuffisamment drainées), assure, pour le moment, à la fois un espace archéologique, c'est-à-dire une vue approchée des vestiges retrouvés, avec des zones vérifiables pour tout archéologue d'aujourd'hui ou de demain et une pédagogie publique qui se traduit par des cartels d'explications, des éléments restitués, des effets 
lumineux (comme cet escalier disparu au profil restitué par un verre optique). Le marquage par des matériaux sur le sol supérieur de la dalle permet en outre, d'établir un lien entre réalité urbaine et visite muséographique. Il faut accompagner tout en laissant découvrir. Le travail sur la lumière reste essentiel pour cela. Ici comme en d'autres cas, l'implication de l'archéologue et de son équipe, au-delà du travail de fouille de terrain, est indispensable pour transmettre dans les meilleures conditions une recherche non réservée aux initiées mais qui appartient à tous.

\section{NOTES}

1. SAPIN C., (dir.), Avant-nefs et espaces d'accueil dans l'église entre le IV et le XII siècle : actes du colloque international du CNRS, Auxerre, 17-20 juin 1999, Paris, CTHS, 2002, 508 p.

SAPIN C., (dir.), Archéologie et architecture d'un site monastique. 10 ans de recherche à l'abbaye SaintGermain d'Auxerre, Paris, CTHS, 2000, 493 p.

SAPIN C., (dir.), Peindre à Auxerre au Moyen Âge, IX $X^{e}$-XIV siècles. 10 ans de recherche à l'abbaye SaintGermain d'Auxerre et à la cathédrale Saint-Étienne d'Auxerre, Paris, CTHS, 1999, 312 p.

\section{INDEX}

Index géographique : France/Auxerre

Mots-clés : crypte archéologique 